\title{
Condiciones de salud mental durante la pandemia por COVID-I9
}

\author{
Silvia Morales Chainé', Alejandra López Montoya', Alejandro Bosch Maldonado', Ana Beristain Aguirre', \\ Rebeca Robles García ${ }^{2}$, Fuensanta López Rosales ${ }^{3}$, Carmen Fernández Cáceres ${ }^{4}$ \\ I Universidad Nacional Autónoma de México \\ 2 Instituto Nacional de Psiquiatría "Ramón de la Fuente Muñíz" \\ ${ }^{3}$ Universidad Autónoma de Nuevo León \\ ${ }^{4}$ Centros de Integración Juvenil, A. C.
}

\section{RESUMEN}

Introducción: las condiciones por la pandemia del COVID-19 promueven riesgos a la salud mental. Objetivo: se evaluó el nivel de riesgo a desarrollar condiciones de salud mental en función de las situaciones relacionadas con el COVID-19: el confinamiento, síntomas o sospecha de COVID-19, el fallecimiento de personas cercanas, el uso de alcohol y la violencia en el hogar. Método: participaron 8,348 personas de origen mexicano, con un promedio de 25 años ( $D E=11.24), 50 \%$ solteros $(4,133)$, $69 \%$ mujeres $(5,720), 47 \%$ en cuarentena $(3,948), 50 \%$ parcialmente en cuarentena $(4,193)$ y $3 \%$ que no estaba en cuarentena (207). El estudio fue correlacional predictivo ex post facto utilizando la WebApp del Cuestionario para la detección de riesgos a la Salud Mental COVID-19 (Alfa de Cronbach: 0.96; varianza explicada del $64 \%$ ) programado con Linux $\AA, \mathrm{PHP} \circledast, \mathrm{HTML} \circledast, \mathrm{CSS} \AA$, y JavaScript ${ }^{\circledR}$. Las variables latentes se derivaron de 124 iteraciones con 103 parámetros $(t[496]=201,464$, $p=.000$ ), un $C F I=0.921$, un $T L I=0.908$, un $R M S E A=$ $0.067(0.066-0.068)$ y un $S R M R=0.051$. Resultados: la evitación predijo el estrés agudo, éste predijo la ansiedad de salud; la ansiedad por salud predijo la ansiedad generalizada/tristeza y la somatización; y estas últimas, el distanciamiento/enojo en las personas. Discusión y conclusiones: el confinamiento, la sospecha o tener síntomas, el consumo explosivo de alcohol y reportar violencia emocional o física, se asociaron con los riesgos a la salud mental. Estudios posteriores evaluarán el impacto de estrategias de atención a distancia sobre los riesgos de estrés agudo y postraumático.

Palabras clave: salud mental, impacto COVID-19, ansiedad, somatización, violencia.

\begin{abstract}
Introduction: the conditions of the COVID-19 pandemic promote mental health risks. Objective: the goal of the study was to assess the risk of developing mental health conditions as a result of COVID-19 situations: containment, symptoms or COVID-19 suspected, death of near people, use of alcohol, and violence. Method: it participated 8,348 Mexican, with a mean of 25 years old ( $S D=11.24), 50 \%$ single $(4,133), 69 \%$ women $(5,720)$, $47 \%$ in quarantine $(3,948), 50 \%$ partially in quarantine $(4,193)$, and $3 \%$ that they were not in quarantine (207). The kind proposed was a predictive correlational study using the WebApp application of the Questionnaire of Mental Health Screening in COVID-19 (Alpha= 0.96; 64 of explained variance) programed by Linux $\AA$, PHP $\AA$, HTML $\AA$, CSS $\AA$, and JavaScript $($ ). Latent variables derivate of 124 iterations with 103 parameters $(t[496]=$ 201,464, $p=.000$ ), a $C F I=0.921$, a $T L I=0.908$, a RMSEA $=0.067(0.066-0.068)$, and a $S R M R=0.051$. Results: results showed that avoidance predicted acute stress, this predicted anxiety related to health; anxiety related to health predicted generalized anxiety/sadness and somatization; and generalized anxiety/sadness predicted, disengagement/anger in people. Discussion and conclusions: containment, symptoms, or suspected COVID-19, explosive use of alcohol or reporting of emotional or physical violence, were associated with the risk of mental health conditions. Next studies should assess the impact of distance psychological interventions over the risk of developing acute and posttraumatic stress.
\end{abstract}

Keywords: mental health, COVID-19 impact, anxiety, somatization, violence.

\footnotetext{
Autor de correspondencia:

Silvia Morales Chainé: smchaine@gmail.com

Recibido: 12 de junio de 2020

Aceptado: 22 de septiembre de 2020

doi: 10.28931/riiad.2020.2.03
} 


\section{INTRODUCCIÓN}

El riesgo de contraer el virus SARS-CoV-2 surgió para la especie humana a finales del 2019, en Hubei, China, y paulatinamente se diseminó alrededor del mundo. Para el 4 de junio de 2020, se reportaron a nivel mundial 7,312,198 personas diagnosticadas con COVID-19; 413,003 personas fallecidas; y 3,598,522 personas recuperadas (Organización Mundial de la Salud [OMS], 2020). La enfermedad de las vías respiratorias por contagio del COVID-19 tiene una tasa de mortalidad alta, con especial afectación en las personas con padecimientos previos, como diabetes, y entre particulares grupos etarios (Yang et al., 2020). Al momento señalado, en México existían 124,301 casos acumulados de personas con diagnóstico positivo por COVID-19; 18,904 activos, 90,748 casos recuperados; 14,649 personas fallecidas y un índice de letalidad en México de 11.8\% (Secretaría de Salud, 2020).

Ante el riesgo de padecer COVID-19 y la amenaza real por esta enfermedad, existe una serie de estrategias de afrontamiento eficiente para prevenir y promover la salud mental de la población (Xiang et al., 2020). Por lo tanto, además de los riesgos a la salud física por la pandemia del COVID-19, existe un amplio margen de condiciones asociadas con la salud mental de las personas (Zandifar \& Badrfam, 2020) que los profesionales deben considerar. Resulta fundamental evaluar y manejar crisis psicológicas, conductas impulsivas y de riesgo en la población, para prevenir crisis de ansiedad y problemas emocionales, además de reducir el estrés durante la pandemia por el COVID-19 (Chen et al., 2020). En particular, existe un conjunto de estrategias requeridas para enfrentar el estrés agudo, la ansiedad, la tristeza profunda, el uso explosivo de alcohol, la violencia y otras condiciones de salud mental, asociadas a la pandemia que se vive en el mundo (Zhue et al., 2020).

Es fundamental evaluar los riesgos a la salud mental en los primeros estadíos de cualquier evento traumatizante como lo es pandemia por COVID-19. Dentro de los riesgos esperados se encuentra el estrés agudo y las condiciones emocionales (Yang et al., 2020). El estrés constituye una respuesta de adaptación del organismo que aumenta las probabilidades de enfrentar situaciones adversas de manera efectiva. Se caracteriza por respuestas fisiológicas, motoras, conductuales, emocionales y cognoscitivas que resultan en acciones de huida, parálisis o de afrontamiento ante las condiciones identificadas como amenazantes o estresantes (Lazarus \& Folkman, 1984; 1986). Sin embargo, el grado de riesgos generalmente rebasa psicológica y emocionalmente la capacidad de las personas, llevándolas a mostrar condiciones de estrés y crisis, entre las que se encuentran la fatiga, los problemas de sueño, la irritabilidad, los problemas de atención y el miedo, que pueden llegar hasta el consumo de sustancias y la violencia (Li, Ge et al., 2020).

En el caso particular del riesgo a padecer COVID-19, $\mathrm{Li}, \mathrm{Ge}$ y colaboradores (2020) evaluaron los riesgos a la salud mental en 740 personas por medio de una escala de trauma vicario (McCann y Pearlman, 1990), vía una WebApp, durante la primera semana de cuarentena en China. Los investigadores indicaron la existencia de niveles de trauma vicario entre la población estudiada, es decir, respuestas fisiológicas, psicológicas, emocionales, conductuales y cognitivas que pueden manejarse de forma correcta bajo los lineamientos de prevención de contagio que se toman en los países. De Camargo (2010) señalaba que las personas responden a un evento estresante o traumático en función de un síndrome general de adaptación, caracterizado por una fase de alarma, otra de resistencia y una de agotamiento. La primera fase se caracteriza por un estado de alerta, pero de congelamiento de la acción ante el evento estresante, en el cual se activa un mecanismo de atención y evaluación del suceso traumático; en este periodo, las personas pueden responder de forma poco eficiente durante el evento. La fase de resistencia se caracteriza por el nivel de estrés que mantiene activo al organismo durante semanas, meses y hasta años, y que, si tiene una corta duración, le permite retornar a su equilibrio fisiológico y establecer una condición de vida cotidiana fisiológicamente normal. Por último, la fase de agotamiento se caracteriza por cansancio, ante la presentación repetida del evento estresante, que puede asociarse con la presencia de insomnio (Xiang et al., 2020), fatiga, falta de concentración, reacciones cardiovasculares, metabólicas, endócrinas, problemas emocionales, gastrointestinales, e infartos cardiacos o cerebrovasculares, entre otros riesgos (Lazarus \& Folkman, 1984; 1986; Moos, 1995).

$\mathrm{Li}$, Wang et al. (2020) valoraron las emociones negativas (ansiedad, depresión, indignación, felicidad) y cognitivas (juicio de riesgo social y satisfacción de vida) dentro de las tres primeras semanas de la cuarentena por COVID-19, con la participación de 17,865 personas, de las cuales $75 \%$ eran mujeres, entre los ocho y 56 años $(M=33)$. La evaluación se llevó a cabo a través del análisis de las preocupaciones por medio de las expresiones lingüísticas, antes y después del riesgo sanitario por COVID-19, y por medio de un cuestionario en plataforma Web. Los autores concluyeron que existía un aumento en los reportes de emociones negativas (ansiedad, depresión e indignación), así como un decremento en las emociones positivas (felicidad), entre el antes y después del riesgo a padecer COVID-19. En cuanto a los indicadores cognitivos, las actitudes negativas aumentaron y los niveles de satisfacción de vida disminu- 
yeron, en un marco en el que las actividades familiares y de salud parecían haber aumentado y las actividades recreativas y sociales, haber disminuido. Dicha evidencia mostró que las condiciones de riesgo derivaron en respuestas emocionales relacionadas con el estrés cargado de ansiedad y emociones negativas; los autores también concluyeron que las conductas de cohesión y apoyo social pueden regular los efectos negativos derivados de una condición estresante o traumática como la pandemia por COVID-19.

En otro estudio reciente, Morales-Chainé, Palomero et al. (en prensa) evaluaron el nivel de estrés, emociones y pensamientos negativos, durante el primer mes de contingencia sanitaria por COVID-19 en México, en función de las variables sociodemográficas. Trabajaron con 1,906 personas mexicanas entre los 11 y 69 años de edad $(M=$ 29; $D E=9.53)$, de los cuales $65 \%$ reportó estar en cuarentena, mismos que respondieron el Cuestionario de Tamizaje de Salud Mental en Emergencias (Cuevas et al., 2018; a través de una WebApp para Android $\AA$, iOS $\AA$, Symbian $\AA$, Windows $\AA$, WebOS $\AA$ ). Los autores concluyeron que las personas que se encontraban en cuarentena tuvieron más pérdida de interés, deseos de hacerse daño, dificultad para dormir y poner atención, que aquellas que no se encontraban en confinamiento; asimismo, reportaron que las mujeres en condición de cuarentena, mostraron más estrés agudo que los varones.

En otro estudio, Morales-Chainé, López et al. (en prensa) evaluaron el nivel de riesgo a la salud mental durante la segunda fase de la contingencia sanitaria por COVID-19 en México, en función del confinamiento, del uso de alcohol y la violencia en el hogar; valoraron los riesgos a la salud mental con 9,361 personas mexicanas a través de una WebApp. Los autores concluyeron que, durante la fase dos de la pandemia, la evitación, la tristeza, el distanciamiento, el enojo y la ansiedad generalizada estuvieron predichos por el nivel de estrés agudo, y este último por la ansiedad relacionada con la salud. Quienes se encontraban en cuarentena, también consumieron alcohol de forma explosiva y vivieron violencia emocional o física en mayor medida que los que no se encontraban en confinamiento.

De este modo, las personas pueden reportar emociones negativas (aversión, ansiedad; Mortensen et al., 2010), así como una evaluación cognitiva negativa ( $\mathrm{Li}$, Wang et al. 2020). Parece ser que la presencia del riesgo a padecer COVID-19 constituye un evento extremo similar a otros eventos traumáticos que puede producir estrés agudo (como los descritos por Carlson, 2014). Es decir, la pandemia por COVID-19 parece ser un evento que se asocia con el desarrollo del trastorno por estrés postraumático (TEPT; APA, 2013); Tales condiciones están caracterizadas por reportes de re- cuerdos o pensamientos reiterativos del evento disparador que pueden evidenciarse a través de pesadillas, miedo intenso y pensamientos recurrentes automáticos involuntarios del suceso traumático. Las condiciones de salud mental asociadas al riesgo a padecer COVID-19 también se caracterizarían por condiciones de evitación deliberada de pensamientos, recuerdos, actividades o situaciones que derivarían en el recuerdo del evento estresante. Ante la contingencia sanitaria por este nuevo virus, parecen existir sensaciones de amenaza inmediata caracterizada por la hipervigilancia o reacciones emocionales elevadas. Estos estados suelen ser temporales en una condición de estrés agudo, en la mayoría de las personas, pero en una condición como la del riesgo a padecer COVID-19, se podría convertir en crónica como lo es el TEPT, en el cual las reacciones cognitivas, emocionales y conductuales permanecerían a lo largo del tiempo y derivarían en otras condiciones de salud física y mental.

En un estudio reciente, Ho et al. (2020) indicaron que $54 \%$ de las personas en condición de cuarentena por COVID-19, reportaron un impacto psicológico entre moderado y severo; $16.5 \%$ reportaron síntomas depresivos de moderados a severos: $29 \%$ síntomas de ansiedad de moderados a severos, y $8 \%$ niveles de estrés de moderados a severos. La exposición al riesgo a padecer coronavirus parece generar ansiedad por el riesgo a enfermar o morir, desesperanza, culpa por que otros enfermen y su asociación con otras condiciones de riesgo a la salud mental, como el uso de alcohol y la violencia (Morales-Chainé, Palomero et al., en prensa). En concreto, la presencia de otros riesgos asociados como el uso de sustancias psicoactivas o ser víctima de violencia, pueden favorecer la evolución de las situaciones de alarma a la salud mental (Organización Panamericana de la Salud [OPS], 2017; Yang et al., 2020).

El objetivo del presente trabajo consistió en evaluar el nivel de riesgo a desarrollar condiciones de salud mental en función de las situaciones relacionadas con el COVID-19: el confinamiento, síntomas o sospecha de la enfermedad, las pérdidas de personas cercanas, el uso de alcohol y la violencia en el hogar; similares a los observados ante otros eventos amenazantes para la vida de las personas. El estudio permitió determinar las variables latentes de estrés agudo, distanciamiento/ enojo, ansiedad generalizada/tristeza, ansiedad asociada a la salud física, somatización y evitación, ante las situaciones asociadas con la pandemia por COVID-19, las pérdidas de personas cercanas, el reporte de consumo de alcohol y la presencia de violencia en el hogar; condiciones que representan el nivel de impacto de la pandemia sobre la salud mental en la población en México. 


\section{MÉTODO}

\section{Participantes}

El estudio incluyó a 8,348 personas (ver Tabla 1), con un promedio de 25 años de edad ( $D E=11.24)$ en un rango de 12 a 74 años, 50\% solteros (4.133) y 69\% mujeres $(5,720)$. Los participantes respondieron el instrumento por invitación, de forma voluntaria, por lo que los grupos de investigación no pudieron ser homogéneos en cantidad o en condición de cuarentena. Es decir, 61\% de las personas $(5,071)$ trabajaba al momento de la colaboración, 47\% reportó encontrarse en cuarentena $(3,948)$, $50 \%$ se encontraba parcialmente en cuarentena $(4,193)$ y $3 \%$ no estaba en cuarentena (207). El 10\% (817) de las personas refirió síntomas o sospecha de padecer COVID-19 al momento de responder y 5\% (432) reportó pérdidas de familiares o personas cercanas.

Las personas respondientes aceptaron las políticas de privacidad, confidencialidad y manejo de la información mediante el consentimiento informado, en el que se explicó que la confidencialidad de la información se mantendría a través de la utilización de promedios grupales, que los resultados del estudio se utilizarían con fines de investigación epidemiológica y difusión de re- sultados; se definió la duración de su participación y se especificó que tenían derecho a declinar el uso de su información y participación en cualquier momento del estudio. No se entregaron incentivos, pero se programó una retroalimentación inmediata en la cual se daba acceso a recursos de psicoeducación (infografías, videos y cursos en plataforma Moodle $₫$ sobre el COVID-19, de autocuidado y relajación) y la disponibilidad de un número telefónico de atención psicológica a distancia, indicándole los beneficios de ingresar o llamar para prevenir condiciones de salud mental. El protocolo fue aprobado por el Comité de Ética de Investigación del Instituto Nacional de Psiquiatría Ramón de la Fuente Muñiz (INPRFM) el 16 de abril de 2020.

\section{Instrumentos}

Para efectos del estudio, se utilizó el Cuestionario para la detección de riesgos a la Salud Mental COVID-19 (basado en Cuevas et al., 2018; Goldberg et al., 2017; González, 2015; Velasco et al., 2006; Arrieta et al., 2017; Arroll et al, 2010; Mitchel et al., 2016; Morales-Chainé et al., 2019; Morales-Chainé, Palomero et al., en prensa), programado en una plataforma (https://www.misalud. unam.mx/covid19/ desarrollada en Linux ${ }^{\circ}, \mathrm{PHP} \circledast$,

Tabla 1

Número de personas respondientes de la población general, en función de su condición relacionada con el COVID-19, si tenían pérdidas cercanas, confinamiento y género

\begin{tabular}{|c|c|c|c|c|c|c|c|}
\hline \multirow{2}{*}{ Confinamiento } & \multirow{2}{*}{ Sexo } & \multicolumn{2}{|c|}{$\begin{array}{l}\text { Sin condición } \\
\text { COVID-19 }\end{array}$} & \multicolumn{2}{|c|}{$\begin{array}{c}\text { Con alguna condición } \\
\text { COVID-19 }\end{array}$} & \multirow[b]{2}{*}{ Subtotal } & \multirow[b]{2}{*}{ Total } \\
\hline & & $\begin{array}{c}\text { Sin } \\
\text { pérdidas }\end{array}$ & $\begin{array}{c}\text { Con } \\
\text { Pérdidas }\end{array}$ & $\begin{array}{c}\text { Sin } \\
\text { pérdidas }\end{array}$ & $\begin{array}{c}\text { Con } \\
\text { Pérdidas }\end{array}$ & & \\
\hline \multirow[t]{2}{*}{ Sí } & Mujeres & 2578 & 98 & 241 & 29 & 2946 & \multirow{2}{*}{3948} \\
\hline & Hombres & 879 & 30 & 67 & 26 & 1002 & \\
\hline \multirow[t]{2}{*}{ Parcialmente } & Mujeres & 2314 & 92 & 173 & 67 & 2646 & \multirow{2}{*}{4193} \\
\hline & Hombres & 1317 & 50 & 165 & 15 & 1547 & \\
\hline \multirow[t]{2}{*}{ No } & Mujeres & 104 & 4 & 9 & 11 & 128 & \multirow{2}{*}{207} \\
\hline & Hombres & 61 & 4 & 8 & 6 & 79 & \\
\hline \multirow[t]{2}{*}{ Subtotal } & Mujeres & 4996 & 194 & 423 & 107 & 5720 & \\
\hline & Hombres & 2257 & 84 & 240 & 47 & 2628 & \\
\hline \multirow[t]{2}{*}{ Subtotal } & & 7253 & 278 & 663 & 154 & & \\
\hline & & \multicolumn{2}{|c|}{7531} & \multicolumn{2}{|c|}{$81 \overline{7}$} & & \\
\hline Total & & & & 8,348 & & & \\
\hline
\end{tabular}


HTML ${ }^{\circledR}$, CSS $\circledast$, y JavaScript $($ ) que encripta la información bajo los estándares de seguridad informática de las leyes mexicanas para la protección de datos personales. El cuestionario está integrado por cuatro secciones: 1) Condición por COVID-19 (un reactivo de síntomas y un reactivo de pérdidas); 2) Datos socioeconómicos (por ejemplo con quién vive, dónde, condición por cuarentena, perfil, sexo, edad, estado civil, escolaridad, ocupación, entidad federativa, instancia de salud y condición de salud física); 3) Condiciones de salud mental (32 reactivos que evalúan: estrés agudo, distanciamiento/enojo, ansiedad generalizada/tristeza, de salud, somatización y evitación; con una escala de respuesta del cero al 10, donde cero representa: "nada" y 10 representa "extremadamente describe lo que siento"; lo cual se transforma en porcentaje promedio de respuesta); y 4) Uso de bebidas alcohólicas y conductas violentas: consumo explosivo de alcohol (un reactivo con tres opciones de respuesta); y violencia (dos reactivos con opción de respuesta dicotómica: si o no). El cuestionario tiene un alfa de Cronbach de 0.96 , comunalidades mayores al 0.30 para todos los reactivos, una varianza explicada del $64 \%$. En la evaluación del estado de salud mental, en la Figura 1 se representa la conformación de las seis variables latentes: estrés agudo (siete reactivos), con 92 iteraciones y 32 parámetros (t[21] $=33,599.183, p=0.000)$, un $C F I=1.000, T L I=1.000, R M-$ $S E A=0.000(0.000-0.004)$ y un $S R M R=0.000$; distanciamiento/enojo (siete reactivos), con 93 iteraciones y 31 parámetros (t[21] $=27,612.054, p=0.000)$, un $C F I=1.000$, $T L I=1.000, R M S E A=0.000(0.000-0.014)$ y un $S R M R$ $=0.002$; ansiedad generalizada/tristeza (seis reactivos), con 66 iteraciones y 25 parámetros $(t[15]=45,140.511$, $p=0.000$ ), un $C F I=1.000, T L I=1.000, R M S E A=0.004$ (0.000-0.022) y un $S R M R=0.001$; ansiedad respecto a la salud (cuatro reactivos), con 40 iteraciones y 14 parámetros (t[6] $=23,162.738, p=0.000$ ), un $C F I=1.000, T L I=$ 1.000, RMSEA $=0.000$ (0.000-0.000) y un $S R M R=0.000$; somatización: (cinco reactivos), con 50 iteraciones y 18 parámetros (t[10] $=12,841.131, p=0.000)$, un $C F I=1.000$, $T L I=1.000, R M S E A=0.000(0.000-0.013)$ y un $S R M R=$ 0.001; evitación: (tres reactivos), con 33 iteraciones y 9 parámetros (t[3] $=4,591.330, p=0.000)$, un $C F I=1.000, T L I$ $=1.000, R M S E A=0.000(0.000-0.000)$ y un $S R M R=0.000$.

Adicionalmente, la plataforma misalud.unam. $\mathrm{mx} / \mathrm{co}-$ vid19/: 1) facilita la inmediata derivación con la o el especialista; 2) calcula datos estadísticos anónimos para monitorear la evolución de la contingencia en términos de su impacto en la salud mental de la población; 3) administra la operación de las actividades de monitoreo o suspensión; y 4) registra la supervisión de la atención psicológica (ficha de datos generales, resultados del cuestionario, plan de servicios, resúmenes de sesión, registro de egreso y seguimiento).

\section{Procedimiento}

Se utilizó un estudio correlacional predictivo, en el cual, a través de la página oficial de la Secretaría de Salud Federal en México y de una universidad pública en México, se convocó a la comunidad universitaria y abierta que necesitara servicio psicológico a ingresar a la dirección electrónica misalud.unam.mx/covid19/ y responder el cuestionario, durante la Fase 3 de la cuarentena (entre el 21 de abril y el 15 de mayo de 2020). Las personas participantes leyeron las siguientes instrucciones:

El riesgo a padecer COVID-19, constituye una condición social sin precedentes que nos afecta a todas y todos. El momento actual nos demanda una forma de enfrentar cómo nos sentimos; como resultado podríamos identificar qué hacer al respecto y dónde podríamos encontrar ayuda profesional con evidencia de éxito que pudiéramos necesitar. En consecuencia, le pedimos complete usted la siguiente información. A partir de lo que responda, recibirá retroalimentación, recomendaciones para actuar ante las emociones, pensamientos y comportamientos que usted pudiera identificar y presentar como resultado de la contingencia de salud que enfrentamos. Su participación es voluntaria y la información que nos proporcione es confidencial. Su manejo se hará con base en la política de privacidad de datos personales.

\section{Análisis de datos}

Para el análisis de los datos se obtuvieron los estadísticos descriptivos (promedios, desviaciones estándar) a través del paquete estadístico IBM $₫$ SPSS $®$, versión 19.0, y la estadística inferencial (Análisis factorial exploratorio, confirmatorio, modelamiento de ecuaciones estructurales y elaboración de gráficos de caja y bigote de la distribución) de los datos, mediante el entorno de programación para análisis de datos RStudio ® versión 1.3.959.

\section{RESULTADOS}

A continuación, se presentan los resultados en función del análisis de los datos. En primer término, se muestran los índices de ajuste del modelamiento de ecuaciones estructurales, para identificar la carga factorial de los reactivos de cada variable latente, así como la relación predictiva entre las variables. En segundo término, se muestran las distribuciones gráficas de los puntajes obtenidos por las personas participantes en función de la cuarentena y su sexo, así como para cada una de las condiciones de riesgo a la salud mental, en función de 
las situaciones relacionadas con el COVID-19: síntomas o sospecha de enfermedad, pérdida de alguna persona cercana, uso explosivo de alcohol y ser víctima de violencia.

En la Figura 1 se representan las variables latentes en función de su carga factorial producto del modelamiento de ecuaciones estructurales, derivada de 124 iteraciones con 103 parámetros ( $t[496]=201,464.023, p=.000)$, un $C F I=0.921$, un $T L I=0.908$, un RMSEA $=0.067(0.066$ - 0.068) y un $S R M R=0.051$. Conforme se observa, los niveles de evitación predicen los niveles de estrés agudo ( $R 2=0.586)$; estos últimos predicen los de ansiedad por la salud $(R 2=0.951)$ y éstos, a su vez, predicen los de ansiedad generalizada/tristeza y somatización ( $R 2=0.830$; y $R 2=0.787$, respectivamente); asimismo, la ansiedad generalizada/tristeza predice los niveles de distanciamiento/enojo $(R 2=0.942)$.

En la Figura 2 se representa la distribución del puntaje obtenido por las personas en función de los cuartiles para todas las escalas de riesgo a la salud mental: estrés agudo (primer renglón de gráficos), distanciamiento y enojo (segundo renglón de gráficos), ansiedad generalizada/ tristeza (tercer renglón de gráficas) ansiedad por la salud (cuarto renglón de gráficos), somatización (quinto renglón de gráficos) y evitación (sexto renglón de gráficos) en función de la condición respecto a la cuarentena por el COVID-19 (sí, parcialmente, o no está en cuarentena; columna izquierda de gráficos) y sexo de los participantes (columna derecha de gráficos). Un análisis multivariado indicó que: 1) hubo diferencias estadísticamente significativas $(F[2,8,346]=4,79, p=0.008)$ entre los niveles de estrés agudo en función de la condición por contingencia: sí ( $M=41.21 ; D E=26.72)$, parcialmente $(M=40.38 ; D E=26.99)$ y no está en confinamiento $(M$ = 35.54; $D E=31.42)$; 2) hubo diferencias estadísticamente significativas $(F 2,8,346]=41.93, p=0.000)$ entre los niveles de distanciamiento/enojo en función de la condición por contingencia: sí $(M=40.96$; $D E=27.03)$, parcialmente $(M=35.99 ; D E=25.95)$ y no está en confinamiento $(M=31.75 ; D E=29.06)$; 3 ) hubo diferencias estadísticamente significativas $(F 2,8,346]=40.78, p=$ 0.000 ) entre los niveles de ansiedad generalizada/tristeza: sí $(M=47.41 ; D E=32.22)$, parcialmente $(M=41.54$; $D E=31.96)$ y no está en confinamiento $(M=36.00 ; D E$ = 34.38); y 4) hubo diferencias estadísticamente significativas ( $F 2,8,346]=5.83, p=0.003$ ) entre los niveles de ansiedad con relación a la salud en función de la condición por contingencia: sí $(M=44.60 ; D E=32.64)$, parcialmente $(M=42.49 ; D E=32.39)$ y no está en confinamiento $(M=39.43 ; D E=36.89)$. No hubo diferencias significativas entre los niveles de somatización ni evitación en función de las condiciones de confinamiento.

Figura 1

Modelo de ecuaciones estructurales de las condiciones de salud mental: estrés agudo, ansiedad por la salud, ansiedad generalizadal tristeza, somatización, distanciamiento/enojo y evitación, reportados por las personas participantes del estudio y su nivel de predicción.

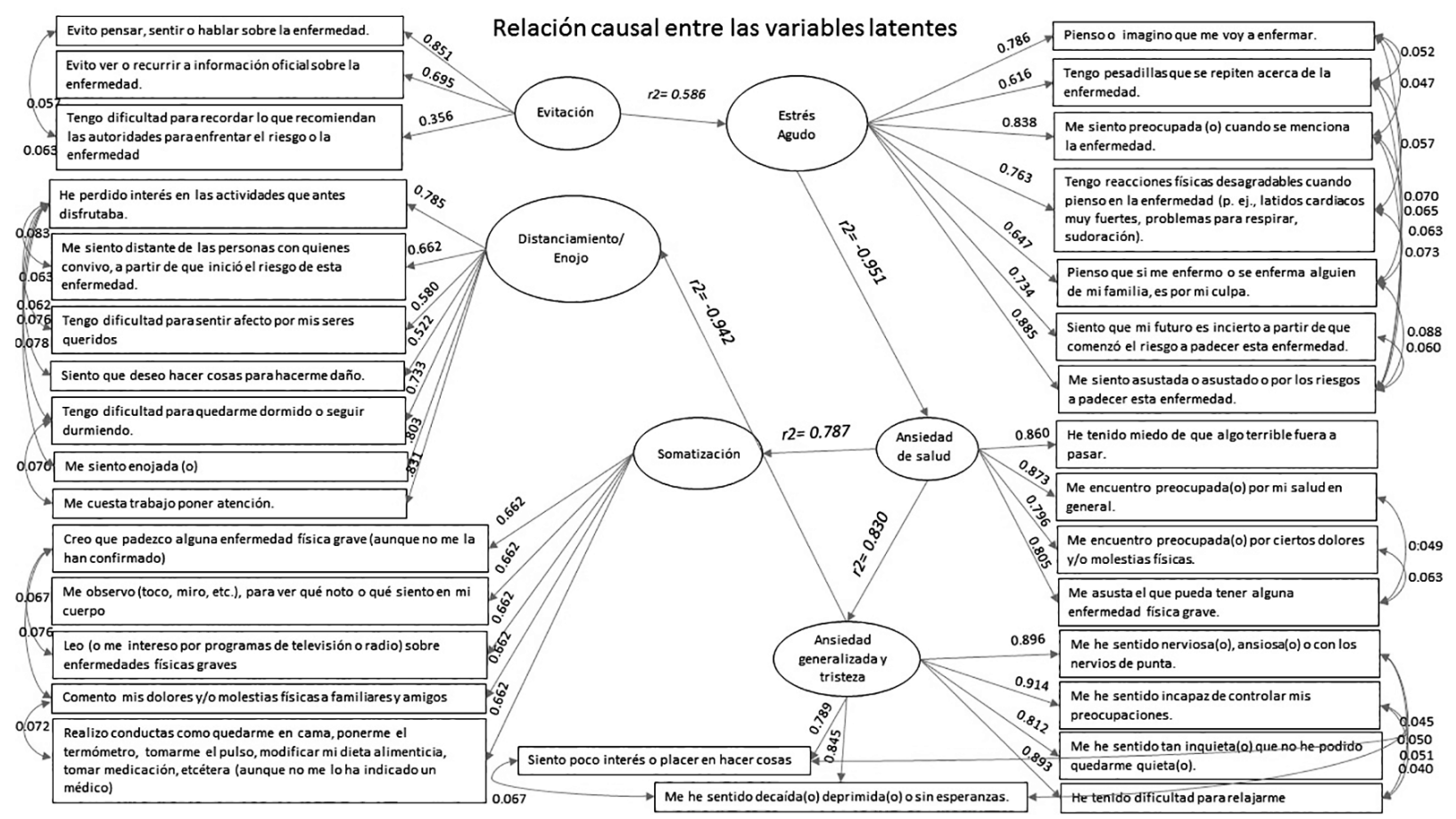


Figura 2

Distribución del puntaje en cuartiles para la escala de estrés agudo (primer renglón de gráficos), distanciamiento/enojo (segundo renglón de gráficos), ansiedad generalizada/tristeza (tercer renglón de gráficos), ansiedad por salud (cuarto renglón de gráficos), somatización (quinto renglón de gráficos) y evitación (sexto renglón de gráficos) en función de la condición de contingencia: Está en cuarentena, parcialmente en cuarentana pues trabaja o sale por víveres o no se encuentra en total cuarentena (columna izquierda de gráficos) y por sexo de las personas participantes (columna derecha de gráficos). Las líneas inferiores y superiores de cada caja representan el percentil $25^{\circ}$ y $75^{\circ}$, respectivamente, y la línea horizontal dentro de cada caja representa el percentil $50^{\circ}$ (mediana grupal). Las líneas verticales extendidas de la caja representan los valores mínimos y máximos que no son outliers.
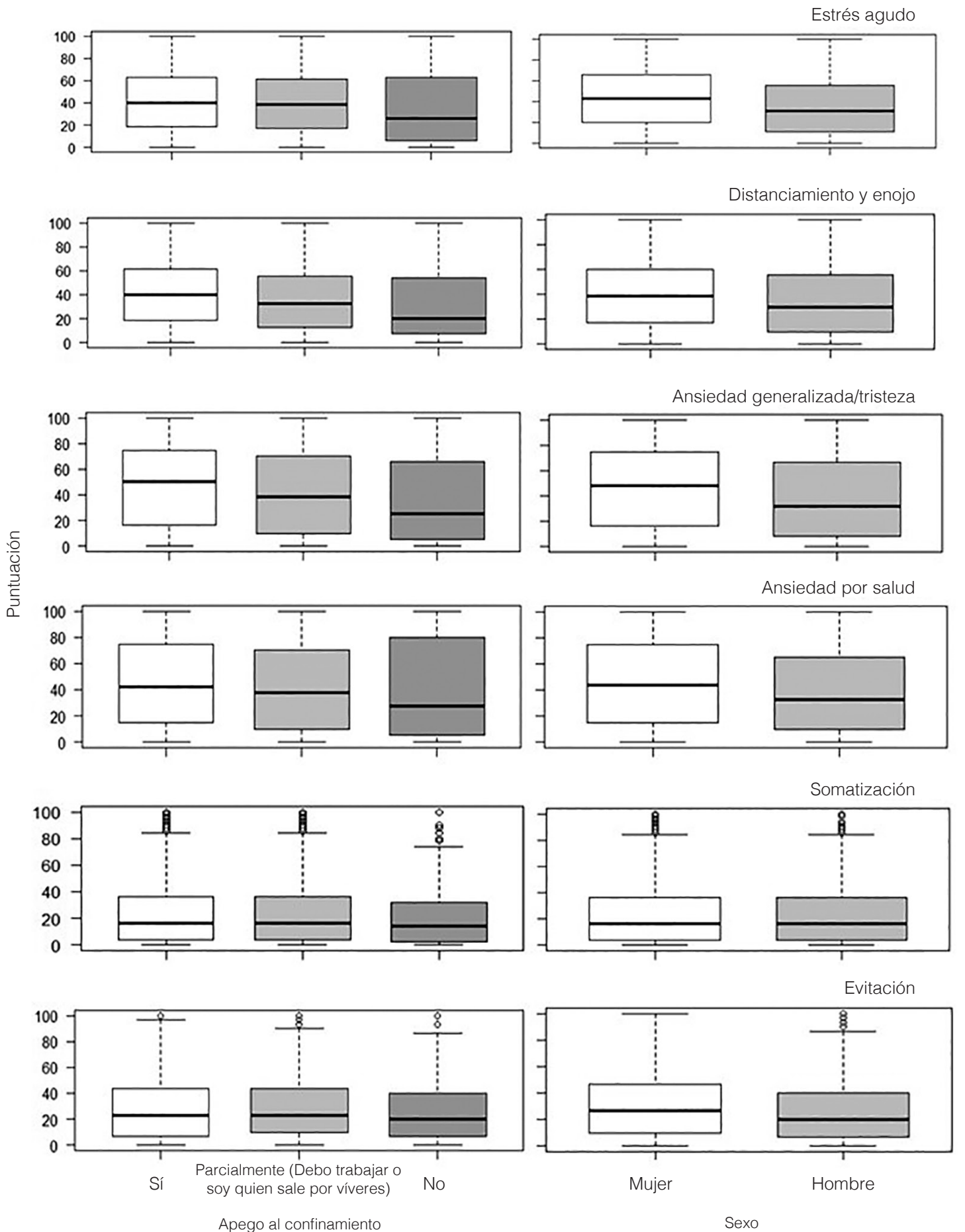
En la misma Figura 2 se representa la distribución por cuartiles en las escalas de salud mental en función del sexo de los participantes. Un análisis multivariado indicó que: 1) hubo diferencias estadísticamente significativas entre: los niveles de estrés agudo $(F 1,8,346]=134.47$, $p=0.000)$ de las mujeres $(M=42.96 ; D E=26.69)$ y los hombres $(M=35.64 ; D E=26.98) ; 2)$ los niveles de distanciamiento/enojo $(F[1,8,346]=79.64, p=0.000)$ de las mujeres $(M=39.99 ; D E=26.36)$ y los hombres $(M=$ 34.41; $D E=26.95)$; 3) los niveles de ansiedad generalizada/tristeza $(F 1,8,346]=150.36, p=0.000)$ de las mujeres $(M=47.09$; $D E=31.95)$ y los hombres $(M=37.84$; $D E=32.15) ; 4)$ los niveles de ansiedad por la salud ( $F 1$, $8,346]=74.64, p=0.000)$ de las mujeres $(M=45.50 ; D E$ $=32.50)$ y los hombres $(M=38.88 ; D E=32.51)$; y 5$)$ los niveles de evitación $(F 1,8,346]=62.84, p=0.000)$ de las mujeres $(M=28.72 ; D E=22.64)$ y los hombres $(M$ $=24.54 ; D E=21.77)$. No hubo diferencias significativas entre los niveles de somatización en función del sexo de las personas.

En la Figura 3 se muestra la distribución por cuartiles de las condiciones de salud: estrés agudo (primer renglón de gráficas), distanciamiento/enojo (segundo renglón de gráficos), ansiedad generalizada/tristeza (tercer renglón de gráficos), por la salud (cuarto renglón de gráficos), somatización (quinto renglón de gráficos) y evitación (sexto renglón de gráficos) en función de la distribución de las personas quienes reportaron: alguna condición relacionada con el COVID-19 (síntomas, sospecha, diagnóstico de la persona respondiente o de un familiar cercano; primera columna de gráficos: 7,531 no tuvieron ninguna condición, y 817 sí tuvieron alguna condición), alguna pérdida familiar o de alguna persona cercana $(7,916$ sin pérdidas y 432 con pérdidas; segunda columna de gráficos), el consumo explosivo de alcohol (tercera columna de gráficas: 1,193 sí consumieron alcohol de forma explosiva; 7,070 no lo consumieron explosivamente; y 85 eligieron la opción de no compartir dicha información); haber sido lastimado(a) emocionalmente (cuarta columna de gráficos: 2,115 reportaron haber sufrido tal violencia y 6,233 no haberla recibido) o físicamente (quinta columna de gráficos: 391 reportaron haberla vivido y 7,957 no). En específico, un análisis multivariado indicó que hubo diferencias estadísticamente significativas entre: 1$)$ los niveles de estrés agudo $(F 1,8,346]=219.76, p=$ $0.000)$ de quienes reportaron alguna condición por $\mathrm{CO}$ VID-19 $(M=53.78 ; D E=27.04)$ y quienes no $(M=39.23$; $D E=26.60) ; 2)$ los niveles de distanciamiento/enojo ( $F 1$, $8,346]=24.95, p=0.000)$ de las personas que reportaron alguna condición por COVID-19 $(M=42.66 ; D E=24.15)$ y las que no $(M=37.76 ; D E=26.89) ; 3)$ los niveles de ansiedad generalizada/tristeza $(F 1,8,346]=91.17, p=$ 0.000 ) de quienes reportaron alguna condición por $\mathrm{CO}$ -
VID-19 $(M=54.37 ; D E=31.16)$ y quienes no $(M=43.07$; $D E=32.23) ; 4)$ los niveles de ansiedad por la salud ( $F 1$, $8,346]=351.81, p=0.000)$ de las personas que reportaron alguna condición por COVID-19 $(M=63.35 ; D E=$ 31.24) y las que no ( $M=41.25$; $D E=32.06)$; 5$)$ los niveles de somatización $(F[1,8,346]=439.55, p=0.000)$ de las personas que reportaron alguna condición por $\mathrm{CO}$ VID-19 $(M=38.87 ; D E=27.44)$ y las que no $(M=21.51$; $D E=21.87) ;$ y 6$)$ los niveles de evitación $(F 1,8,346]=$ 103.15, $p=0.000$ ) de quienes reportaron alguna condición por COVID-19 $(M=34.94 ; D E=23.14)$ y quienes no $(M=26.59 ; D E=22.23)$.

El análisis multivariado también indicó que hubo diferencias estadísticamente significativas entre: 1) los niveles de estrés agudo $(F[1,8,346]=26.53, p=0.000)$ de las personas que reportaron alguna pérdida por $\mathrm{CO}$ VID-19 $(M=47.16 ; D E=29.16)$ y las que no habían perdido a un ser cercano $(M=40.30 ; D E=26.82) ; 2)$ los niveles de distanciamiento/enojo $(F 1,8,346]=5.73, p=0.017)$ de quienes reportaron alguna pérdida por COVID-19 $(M=41.23 ; D E=26.65)$ y quienes no $(M=38.07 ; D E$ $=26.67)$; 3) los niveles de ansiedad generalizada/tristeza $(F[1,8,346]=5.77, p=0.016)$ de las personas que reportaron alguna pérdida por COVID-19 $(M=47.81 ; D E$ = 33.70) y las que no $(M=43.98 ; D E=32.21) ; 4)$ los niveles de ansiedad por la salud $(F 1,8,346]=19.31, p$ $=0.000$ ) de las personas que reportaron alguna pérdida por COVID-19 ( $M=50.13 ; D E=32.27)$ y las que no $(M=43.05 ; D E=32.63) ; 5)$ los niveles de somatización $(F 1,8,346]=18.95, p=0.000)$ de aquellos que reportaron alguna pérdida por COVID-19 $(M=27.91 ; D E=25.63)$ y los que no $(M=22.96 ; D E=22.89) ;$ y 6$)$ los niveles de evitación $(F 1,8,346]=39.82, p=0.000)$ de las personas que reportaron alguna pérdida por COVID-19 ( $M=34.03$; $D E=23.71)$ y las que no $(M=27.04 ; D E=22.33)$.

Adicionalmente, existen diferencias significativas en los niveles de estrés agudo en función de haber reportado el consumo explosivo de alcohol (cinco o más cervezas o copas de vino o tragos "derechos" de alcohol; o bebidas preparadas [tequila, whiskey, mezcal, ron, etc.] en menos de dos horas; $F[2,8,346]=23.24, p=0.000$ ). Las personas que reportaron haber consumido alcohol de esta manera mostraron niveles promedio más altos de estrés agudo $(M=45.45, D E=27.79)$, que las que no lo hicieron $(M=39.91, D E=26.68)$; cabe señalar que las que decidieron no compartir dicha información $(n=85)$ mostraron un promedio bajo de dicho estrés $(M=35.44$, $D E=33.54)$. También hubo diferencias estadísticamente significativas en los niveles de distanciamiento/enojo en función de haber reportado el consumo explosivo de alcohol $(F[2,8,346]=120.72, p=0.000)$. Las personas que reportaron haberlo consumido de esa forma mostraron niveles promedio más altos de distanciamiento/enojo 
$(M=49.18, D E=28.38)$, que las que no lo hicieron $(M=$ 36.38, $D E=25.86)$; nuevamente, quienes decidieron no compartir dicha información mostraron un promedio bajo de estas condiciones $(M=38.35, D E=31.23)$. También hubo diferencias significativas en los niveles de ansiedad generalizada/tristeza en función de haber reportado el consumo excesivo de alcohol $(F[2,8,346]=71.51, p=$ 0.000 ). Las personas que reportaron haberlo hecho mostraron niveles promedio más altos de ansiedad generalizada ( $M=54.43, D E=33.23$ ), que las que no lo hicieron $(M=42.50, D E=31.71)$; las que decidieron no compartir dicha información mostraron un promedio bajo de dicha ansiedad ( $M=40.39, D E=40.25)$. También, se encontraron diferencias significativas en los niveles de ansiedad por salud en función de reportar haber consumido alcohol de forma explosiva $(F 2,8,346]=29.44, p=0.000)$. Las personas que reportaron haberlo hecho mostraron niveles promedio más altos de ansiedad respecto a su salud ( $M$ $=50.01, D E=33.27)$, que las que no lo hicieron $(M=$ 42.37, $D E=32.41$ ); las que decidieron no compartir dicha información mostraron un promedio bajo de ansiedad respecto a su salud ( $M=37.71, D E=32.53)$.

Otro análisis multivariado indicó que existen diferencias significativas en los niveles de somatización en función del consumo explosivo de alcohol $(F 2,8,346]=$ 20.89, $p=0.000$ ). Las personas que reportaron haberlo hecho mostraron niveles promedio más altos de somatización ( $M=26.86, D E=24.37$ ), que las que no lo hicieron $(M=22.68, D E=22.82)$; las que decidieron no compartir dicha información mostraron un promedio bajo de tales condiciones $(M=16.12, D E=17.83)$ Finalmente, otro análisis multivariado indicó que existen diferencias significativas en los niveles de evitación/tristeza en función del consumo explosivo de alcohol $(F 2,8,346]=18.30, p=$ 0.000 ). Quienes reportaron haberlo hecho mostraron niveles promedio relativamente más altos de evitación/tristeza $(M=29.81, D E=24.38)$, que quienes no lo hicieron $(M=$ 26.87, $D E=22.11$ ); sin embargo, quienes decidieron no compartir dicha información mostraron un promedio alto de tales condiciones $(M=37.96, D E=17.59)$.

Respecto al reporte de las personas sobre la violencia emocional recibida, un análisis multivariado indicó que existen diferencias significativas en los niveles de estrés agudo en función de aquellas(os) que fueron lastimados emocionalmente de forma intencional (por ejemplo, humillaciones, insultos, amenazas, ser ignorado, recibir prohibiciones o ser celada; $F[2,8,346]=172.73, p=0.000$ ). Las personas que reportaron haber sido víctimas de violencia emocional mostraron niveles promedio más altos de estrés agudo $(M=47.25, D E=25.54)$, que las que no la recibieron $(M=38.41, D E=27.11)$. También hubo diferencias significativas en los niveles de distanciamiento/ enojo en función de haber reportado violencia emocional
$(F 2,8,346]=859.66, p=0.000)$. Las que reportaron haberla recibido mostraron niveles promedio más altos de distanciamiento/enojo $(M=52.23, D E=25.63)$, que las que reportaron no haberla recibido $(M=33.49, D E$ $=25.32$ ). Hubo diferencias significativas en los niveles de ansiedad generalizada/tristeza en función de haber reportado violencia emocional $(F 2,8,346]=635.14, p$ $=0.000$ ). Quienes refirieron haberla recibido mostraron niveles promedio más altos de ansiedad generalizada $(M=58.92, D E=29.07)$, que quienes no la recibieron $(M$ $=39.18, D E=31.80$ ). Se encontraron diferencias significativas en los niveles de ansiedad por salud en función de reportar violencia emocional $(F 2,8,346]=216.73, p$ $=0.000$ ). Aquellos que indicaron haberla recibido mostraron niveles promedio más altos de ansiedad respecto a su salud ( $M=52.33, D E=30.88)$, que quienes no la reportaron $(M=40.39, D E=32.68)$. Otro análisis multivariado indicó que hubo diferencias significativas en los niveles de somatización en función de haber reportado violencia emocional $(F[2,8,346]=117.50, p=0.000)$. Las personas que reportaron haberla recibido mostraron niveles promedio más altos de somatización $(M=27.88$, $D E=22.99)$, que las que no la recibieron $(M=21.63, D E$ $=22.87$ ). Otro análisis multivariado indicó que hubo diferencias significativas en los niveles de evitación/tristeza en función de haber reportado violencia emocional $(F[2,8,346]=16.68, p=0.000)$. Las personas que reportaron haberla recibido mostraron niveles promedio más altos de evitación/tristeza $(M=29.13, D E=22.92)$, que las que no la recibieron $(M=26.82, D E=22.26)$.

Por último, respecto al reporte de violencia física recibida, un análisis multivariado indicó que hubo diferencias significativas en los niveles de estrés agudo en función de reportar que alguien le lastimó físicamente de forma intencional (por ejemplo, empujones, golpes, pellizcos, etc.; $F[2,8,346]=16.33, p=0.000$ ). Las personas que reportaron haber sido víctimas de violencia física mostraron niveles promedio más altos de estrés agudo $(M$ $=46.03, D E=27.74)$, que las que no la recibieron $(M=$ 40.39, $D E=26.93$ ). También hubo diferencias significativas en los niveles de distanciamiento/enojo en función de reportar haber sido víctima de dicha violencia física $(F 2$, $8,346]=96.57, p=0.000$ ). Las personas que reportaron haberla recibido mostraron niveles promedio más altos de distanciamiento/enojo $(M=51.10, D E=27.12)$, que las que reportaron no haberla recibido $(M=37.60, D E=$ 26.49). Hubo diferencias significativas en los niveles de ansiedad generalizada/tristeza en función de reportar haber sido víctimas de violencia física $(F 2,8,346]=77.08$, $p=0.000$ ). Las personas que reportaron haberla recibido mostraron niveles promedio más altos de ansiedad generalizada/tristeza $(M=58.12, D E=31.14)$, que las que no la recibieron $(M=43.50, D E=32.20)$. Se encontraron 
Figura 3

Distribución del puntaje en cuartiles para las escalas cuyos análisis multivariados indicaron diferencias significativas en las condiciones de salud mental: estrés agudo (primer renglón), distanciamiento/enojo (segundo renglón), ansiedad generalizada/tristeza (tercer renglón) ansiedad por salud (cuarto renglón), somatización (quinto renglón) y evitación (sexto renglón) en función de tener o no condiciones por COVID-19 (primer columna de gráficos), pérdidas por COVID-19 (segunda columna), del reporte de uso de alcohol (tercer columna), violencia emocional recibida (cuarta columna) y violencia física recibida (quinta columna). Las líneas inferiores y superiores de la caja representan el percentil $25^{\circ}$ y $75^{\circ}$, respectivamente, y la línea horizontal dentro de cada caja representa el percentil $50^{\circ}$ (mediana grupal). Las líneas verticales extendidas de la caja representan los valores mínimos y máximos que no son outliers. Los círculos fuera de los valores mínimos y máximos son outliers.
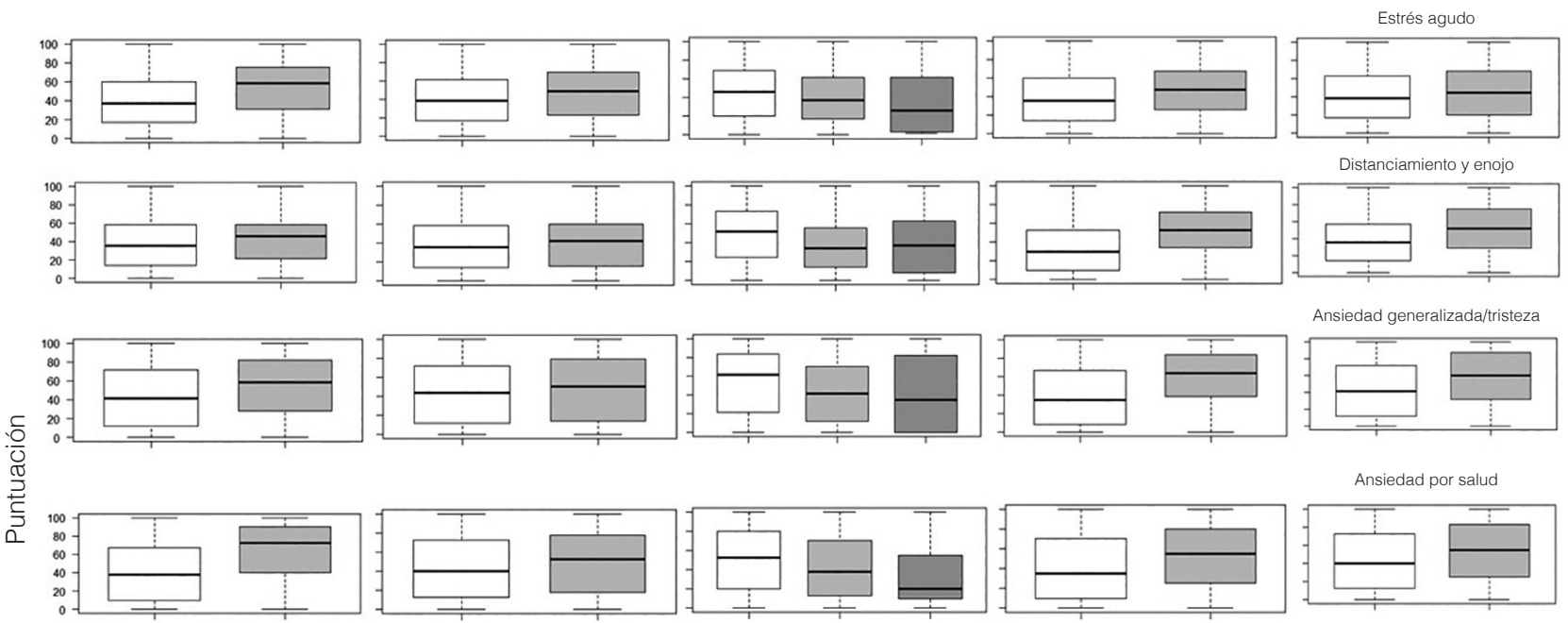

Distanciamiento y enojo

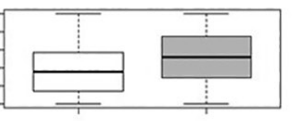

Ansiedad generalizada/tristeza
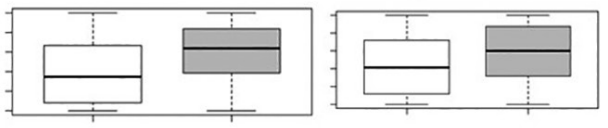

Ansiedad por salud
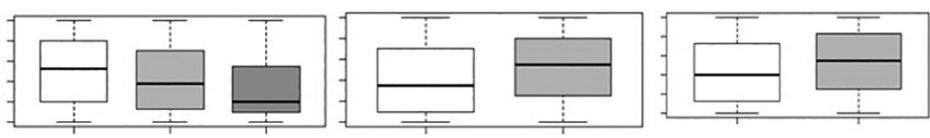

Somatización
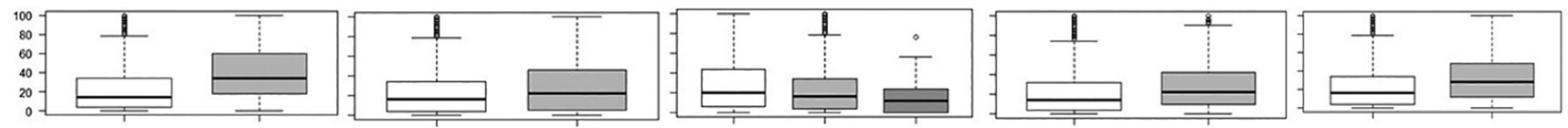

Evitación

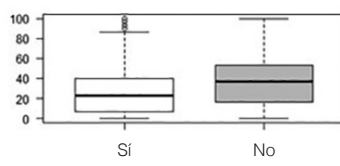

Alguna condición COVID-19

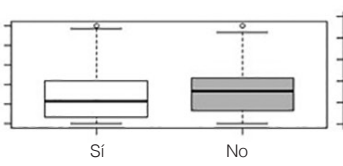

Pérdida por COVID-19

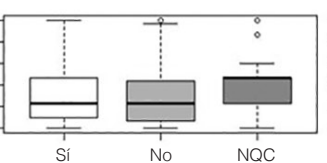

Consume alcohol

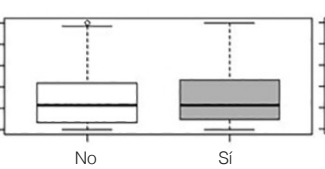

Alguien lastimó emocionalmente a otra persona con la intención insultó, amenazó, ignoró, prohibió, celó).

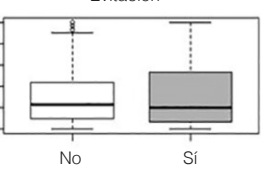

Alguien la (o) lastimó físicamente de forma intencional pellizcar, etcétera). diferencias significativas en los niveles de ansiedad por salud en función de reportar haber sido víctima de violencia física $(F 2,8,346]=38.42, p=0.000)$. Las personas que reportaron haberla recibido mostraron niveles promedio más altos de ansiedad respecto a su salud ( $M$ $=53.38, D E=31.42)$, que las que no la reportaron $(M=$ 42.92, $D E=32.63)$. Otro análisis multivariado indicó que existen diferencias significativas en los niveles de somatización en función de haber sido víctima de violencia física $(F 2,8,346]=65.21, p=0.000)$. Las personas que reportaron haberla recibido mostraron niveles promedio altos de somatización $(M=32.37, D E=25.29)$, que las que no la recibieron $(M=22.76, D E=22.85)$. Un análisis multivariado señaló que hay diferencias significativas en los niveles de evitación/tristeza en función de haber sido víctima de violencia física $(F[2,8,346]=4.01, p=0.045)$. Las personas que reportaron haberla recibido mostraron niveles promedio altos de evitación/tristeza $(M=29.63$, $D E=25.88)$, que las que no la recibieron $(M=27.30, D E$ $=22.27$ ).

\section{DISCUSIÓN}

El objetivo del trabajo fue evaluar el nivel de riesgo a desarrollar condiciones de salud mental en función de las situaciones relacionadas con el COVID-19: la cuarentena, síntomas o sospecha de la enfermedad, las pérdidas de personas cercanas, el uso de alcohol y la violencia 
en el hogar. Se esperaba que las condiciones fueran similares a las observadas ante otros eventos amenazantes para la vida de las personas o las alteraciones traumáticas en general. Así, en la tercera fase sanitaria por COVID-19 en México, los hallazgos indicaron que se encontraron condiciones de estrés agudo, distanciamiento, enojo, ansiedad, tristeza, somatización y evitación, correspondientes a las observadas durante los estadíos avanzados de reacción de las personas ante un evento traumatizante (Zhue et al., 2020). Los hallazgos indicaron que parece existir un conjunto de conductas de evitación, respecto a pensar, sentir, hablar sobre la enfermedad, recurrir a información oficial y para recordar las recomendaciones, que a su vez explicaron los niveles de estrés agudo (Cuevas et al., 2018; Morales-Chainé, López et al., en prensa; Xiang et al., 2020), característicos de los eventos estresantes, fenómenos naturales perturbadores, y sucesos traumáticos. El estrés agudo estuvo caracterizado por el reporte de pensamientos recurrentes de que la persona va a enfermar, por la presencia de pesadillas sobre la enfermedad, preocupación constante, reacciones físicas desagradables, culpa, incertidumbre y miedo (Cuevas et al., 2018; Goldberg et al., 2017; Morales-Chainé, Palomero et al., en prensa). El estrés agudo reportado durante la tercera fase de la contingencia sanitaria, parece haber explicado el reporte de ansiedad relacionada con la salud física, tal como el miedo a lo que le pueda pasar a la persona, preocupación por la salud en general, por ciertos dolores, molestias físicas y el miedo a tener alguna enfermedad grave ( $\mathrm{Li}$ et al., 2020a); así los niveles de estrés predijeron confiablemente la ansiedad por el estado de salud física, lo cual parece provocar niveles de ansiedad generalizada/ tristeza (Zandifar \& Badrfam, 2020), caracterizada por sentirse nervioso, ansioso, incapaz de controlar preocupaciones, inquietud (o) con dificultad para relajarse, con poco interés o placer por hacer cosas, sensaciones de decaimiento, depresión y sentirse sin esperanzas ( $\mathrm{Li}$ et al., 2020a; Xiang et al., 2020); la ansiedad por el estado de salud también parece promover altos niveles de somatización que se caracteriza por pensar en padecer una enfermedad física grave, explorarse físicamente, leer sobre enfermedades graves, comentar los posibles síntomas con otros y quedarse en cama, tomarse la temperatura, tomarse el pulso, automedicarse, etc.; así, la ansiedad por la salud física promueve condiciones más severas de salud mental como la ansiedad generalizada, la tristeza y la somatización. Finalmente, la ansiedad generalizada y la tristeza promovieron comportamientos de distanciamiento y enojo, tales como la pérdida de interés, sentirse distante de los demás, dificultad para sentir afecto, deseo de hacer cosas para hacerse daño, dificultad para dormir, enojo y dificultad para poner atención (Morales-Chaine, Palomero et al., en prensa), nuevamente observando un posible incremento en los grados de severidad desde la ansiedad generalizada hasta el distanciamiento emocional y el enojo caracterizado por la ideación de autolesión y muerte (Zandifar \& Badrfam, 2020).

De acuerdo con los hallazgos del actual estudio, las condiciones de salud mental durante la fase tres de la pandemia por COVID-19 variaron en función del sexo de los participantes de forma similar a lo reportado por Cuevas y colaboradores, 2018 y Morales-Chainé, López y colaboradores (en prensa); de manera que las mujeres mostraron mayor vulnerabilidad a presentar condiciones de estrés agudo, distanciamiento/enojo, ansiedad generalizada/tristeza y ansiedad por la salud física y evitación, que los hombres, ante situaciones en que las percibieron que estas rebasan sus capacidades para afrontar eventos traumáticos.

Adicionalmente, ante las situaciones de orden amenazante o estresante asociadas con la pandemia por COVID-19, como el confinamiento (Zandifar \& Badrfam, 2020), la propia posibilidad de padecer dicha enfermedad, el fallecimiento de familiares directos o cercanos, el consumo explosivo de alcohol o las situaciones de violencia emocional y física en el hogar, las personas del estudio también percibieron mayores niveles de estrés agudo, distanciamiento emocional, enojo, ansiedad generalizada, tristeza, ansiedad por la condición de salud física, somatización y evitación como estrategia de afrontamiento, de forma similar a lo reportado por Li y colaboradores (2020a), cuando se determina la existencia de un trauma vicario, en estos casos por la pandemia por coronavirus.

En general, se puede afirmar que las personas reportaron más estrés agudo, distanciamiento, enojo, ansiedad generalizada, tristeza, ansiedad por la salud, somatización y evitación cuando: se encontraron en confinamiento (Zandifar \& Badrfam, 2020), consumían alcohol de forma explosiva o alguien más había ejercido violencia emocional o física hacia ellas, durante la segunda fase de la contingencia sanitaria por COVID-19. Estos resultados son similares a los síntomas observados en los estudios de Ho y colaboradores (2020), de Mortensen y colaboradores (2010), quienes también reportaron pensamientos negativos (de aversión, ansiedad), y a los de Li, Wang et al. (2020) al encontrar evaluaciones cognitivas negativas ( $p$. ej., ansiedad y tristeza profunda; como lo sugirió Yang et al., 2020) dentro de las semanas posteriores al inicio de la contingencia sanitaria por COVID-19.

Los hallazgos son consistentes con las condiciones de riesgo a desarrollar estrés postraumático, propuestas por De Camargo (2010), quien denominó fase de resistencia 
a aquella caracterizada por las condiciones encontradas en este estudio, donde las personas se mantienen activas en las semanas inmediatas de haber iniciado un evento traumático o estresante, permitiéndoles mantener un cierto nivel de equilibrio fisiológico (Lazarus \& Folkman, 1984; 1986; Moos, 1995); específicamente los hallazgos indicaron una progresión de la evitación como estrategia de afrontamiento hacia la presencia de síntomas por estrés agudo; una transición del estrés agudo hacia niveles de ansiedad por las condiciones de salud mental y hasta la ansiedad generalizada, la tristeza (Zandifar \& Badrfam, 2020) y la somatización; y finalmente, se observó una progresión de la ansiedad generalizada y depresión hasta el distanciamiento y el enojo, todo ello durante la tercera fase de la pandemia por COVID-19. Tales condiciones podrían representar la transición de la fase de alarma, caracterizada por un estado de alerta y evaluación del suceso traumático, a la fase de resistencia, que se distingue por el nivel de estrés que mantiene activo al organismo durante semanas, meses y hasta años y que le permitiría recobrar el equilibrio fisiológico, pero que en condiciones de afrontamiento poco eficiente derivaría hasta la fase de agotamiento, determinada por el cansancio, insomnio, fatiga, falta de concentración, reacciones cardiovasculares, metabólicas, endócrinas, problemas emocionales, gastrointestinales, e infartos cardiacos o cerebrovasculares, entre otros riesgos (Lazarus \& Folkman, 1984; 1986; Moos, 1995). Por ello, estudios adicionales deberán analizar la evolución y progresión de tales condiciones de salud mental (Zandifar \& Badrfam, 2020) hacia la etapa de agotamiento descrita por De Camargo (2019), y con ello el trastorno por estrés postraumático.

Adicionalmente, considérese que el riesgo a afectar la salud mental también podría regularse en función del apoyo familiar (Li, Wang et al., 2020). Aunque los hallazgos indicaron una cierta vulnerabilidad de las mujeres a desarrollar condiciones de salud mental por las situaciones de la pandemia, Morales-Chainé, López et al. (en prensa) ya habían reportado que el mantenerse en confinamiento con familiares podría moderar el efecto de los riegos y amenazas asociados con la pandemia. Por lo tanto, dado que la cohesión y apoyo social pueden regular los efectos negativos derivados de la condición estresante (Zandifar \& Badrfam, 2020), estudios posteriores deberán analizar la relación funcional entre estas variables, comparando la presencia o ausencia de las primeras y su efecto sobre las segundas, en función de las condiciones de la cuarentena sanitaria, las conductas protectoras más que las de riesgo (uso explosivo de alcohol) y la prevención de la violencia en el hogar (Morales-Chainé, Palomero et al., en prensa).

El estrés agudo, distanciamiento, enojo, ansiedad generalizada, tristeza, ansiedad por la salud física, somati- zación y evitación, como las identificadas en el presente estudio, parecen ser el resultado de la contingencia sanitaria por COVID-19, la sospecha o padecerlo, por las pérdidas familiares directas o cercanas o la vulnerabilidad por la condición de ser mujer, pero también de las condiciones de riesgo, como la violencia, o de enfrentamiento, como el consumo explosivo de alcohol. En general, las condiciones de salud mental parecen ser resultado de un evento estresante que puede jugar un papel traumático a mediano plazo, dado que representa un riesgo de vida similar a otros eventos que producen un TEPT (Carlson, 2014). Dado que se observan reacciones emocionales, cognitivas y conductuales negativas en la fase 3 de la contingencia sanitaria, será necesario analizarlas en etapas posteriores a la actual para valorar y prevenir el TEPT, así como para mantener la difusión de estrategias efectivas de prevención e intervención en primer nivel de atención, apoyadas en la evidencia científica (Li, Ge et al., 2020).

Las situaciones asociadas a la pandemia por COVID-19, como el confinamiento, su padecimiento o el fallecimiento de familiares directos o cercanos, parecen constituir eventos traumáticos para las personas, de forma similar a los que se observan ante la ocurrencia de condiciones bélicas, fenómenos perturbadores o de extrema violencia. Las condiciones de estrés y riesgo a la salud mental que le caracterizan, parecen estar constituidos por la evitación, estrés agudo, ansiedad relacionada con la salud, generalizada, tristeza, somatización, el distanciamiento y el enojo (que caracterizan una transición hacia la severidad de síntomas) durante la fase 3 de la pandemia, de forma similar a como Ho et al. (2020) reportaron en las semanas posteriores a esta. Pero, las situaciones de riesgo a la salud mental en este estudio, también parecen explicarse por el uso explosivo de alcohol y las conductas violentas que, junto con la pandemia, podrían estar constituyendo un evento de impacto en la salud mental que merecen ser prevenidos y atendidos de forma eficiente (Yang et al., 2020).

La detección oportuna de riesgos permitirá prevenir condiciones de morbilidad, asociadas con las estrategias de afrontamiento de las situaciones percibidas como amenazantes y que rebasan las capacidades propias. De ello deriva la necesidad de implementar estrategias de manejo e intervención con base en la evidencia empírica (Wang et al., 2020) para reducir los riesgos hacia la salud mental con técnicas a distancia, como lo recomiendan Zhue y colaboradores (2020) y Xiang y colaboradores (2020).

El actual estudio aporta una herramienta de detección temprana y oportuna de riesgos a la salud mental durante la pandemia por COVID-19. Este instrumento constituye uno basado en la implementación a través de 
teléfonos inteligentes que permite evaluar la salud mental, mientras las personas se encuentran en confinamiento, para derivar en el manejo de las condiciones específicas a distancia (como lo recomiendan Xiang et al., 2020 y Zhang et al., 2014). Las recomendaciones puntuales como consecuencia del actual estudio son la atención psicológica a distancia (Zhue et al., 2020), con base en la evidencia científica para enfrentar la ansiedad, el TEPT, el riesgo a recibir violencia o a consumir alcohol.

Dado que la confianza en la autoridad también impacta en la reducción del estrés y la ansiedad, con base en hallazgos como los encontrados en el actual estudio, resultaría fundamental establecer políticas de prevención y promoción de la salud mental a nivel nacional (Yang et al., 2020); a través de estrategias sistemáticas, multi e interdisciplinarias vinculantes entre los niveles de atención. Los hallazgos del actual estudio son congruentes con las recomendaciones de Li y colaboradores (2020b), con respecto a desarrollar una política consistente para informar y apoyarse en los medios de comunicación para promover la salud mental, al tiempo que se previenen los riesgos de contagio y se informa respecto a la evolución del padecimiento.

Estudios posteriores también deberán contribuir con la psicoeducación a distancia para favorecer los estados de salud mental, a través de la comprensión del padecimiento, sus características y su evolución. Evidentemente, las intervenciones deberán moverse en un contexto de atención médica y decisiones político-económicas eficientes para la solución de los problemas asociados al riesgo de enfermar. Es necesario considerar el marco de tiempo en el que se evalúan las condiciones de salud mental, pues las reacciones son variables y se adhieren a las circunstancias sociales del país en cuestión, así como al perfil de las personas beneficiadas y del momento evolutivo del padecimiento.

\section{DECLARACIONES DE LOS AUTORES}

Los autores declaran no tener conflictos de interés.

\section{REFERENCIAS}

American Psychiatric Association (2013). Diagnostic and statistical manual of mental disorders (5th ed.).

Arrieta, J., Aguerrebere, M., Raviola, G., Flores, H., Elliott, P., Espinosa, A., ... \& Palazuelos, D. (2017). Validity and utility of the Patient Health Questionnaire (PHQ)-2 and PHQ-9 for screening and diagnosis of depression in rural Chiapas, Mexico: A crosssectional study. Journal of Clinical Psychology, 73(9), 10761090. doi: 10.1002/jclp.22390
Arroll, B., Goodyear-Smith, F., Crengle, S., Gunn, J., Kerse, N., Fishman, T., Falloon, K., \& Hatcher, S. (2010). Validation of PHQ-2 and $\mathrm{PHQ}-9$ to screen for major depression in the primary care population. The Annals of Family Medicine, 8(4), 348-353. doi: 10.1370/afm.1139

Carlson, N. (2014). Trastorno de ansiedad, trastorno autista, trastorno por déficit de atención con hiperactividad y trastornos por estrés. En N. Carlson (Ed.), Fisiología de la conducta (pp. 626658). Pearson Educación

Chen, Q., Liang, M., Li, Y., Guo, J., Fei, D., Wang, L., He, L., Sheng, C., Cai, Y., Li, X., Wang, J. \& Zhang, Z. (2020). Mental health care for medical staff in China during the COVID-19 outbreak. The Lancet Psychiatry, 7(4), E15-E16. doi: 10.1016/S22150366(20)30078-X

Cuevas, R. C., Cano, R. E. \& Morales-Chainé, S. (2018). Análisis de datos del instrumento de tamizaje (Facultad de Psicología, UNAM). En: Acciones en el marco de la respuesta frente al sismo del 19 de septiembre de 2017. Lecciones aprendidas y buenas prácticas (énfasis en salud mental). Pp. 99-103. SEGOB-CENAPRED.

De Camargo, B. (2010). Estrés, síndrome general de adaptación o reacción general de alarma. Revista Médico Científica, 17(2), 78-86.

Goldberg, D. P., Reed, G. M., Robles, R., Minhas, F., Razzaque, B., Fortes, S., Mari, J. J., Lam, T. P., Garcia J. A., Gask, L., Dowell, A. C., Rosendal, M., Mbatia, J. K., \& Saxena S. (2017). Screening for anxiety, depression, and anxious depression in primary care: A field study for ICD-11 PHC. Journal of Affective Disorders, 213, 199-206. doi: 10.1016/j.jad.2017.02.025.

González Flores, C. (2015). Intervención cognitivo-conductual grupal para disminuir síntomas de depresión, ansiedad y somáticos sin causa médica en pacientes del primer nivel de atención en clínicas del Estado de Jalisco. [Tesis de Maestría en Psicología de la Salud]. Universidad de Guadalajara.

Ho, C. S. H., Chee, C. Y., \& Ho. R. C. M. (2020). Mental health strategies to combat the psychological impact of COVID-19 beyond paranoia and panic. Annals of the Academy of Medicine, Singapure, 49(3), 155-160.

Lazarus, R. S. \& Folkman, S. (1984). El concepto de afrontamiento en estrés y procesos cognitivos. Martínez Roca.

Lazarus, R. S. \& Folkman, S. (1986). Estrés y procesos cognitivos. Martínez Roca

Li, Z., Ge, J., Yang, M., Feng, J., Qiao, M., Jiang, R., Bi, J., Zhan, G., Xu, X., Wang, L., Zhou, Q., Zhou, C., Pan, Y., Liu, S., Zhang, H., Yang, J., Zhu, B., Hu, Y., Hashimoto, K., Jia, Y., ... Yang, C. (2020). Vicarious traumatization in the general public, members, and non-members of medical teams aiding in COVID-19 control. Brain, behavior, and immunity, 88, 916-919. doi: 10.1016/j. bbi.2020.03.007

Li, S., Wang, Y., Xue, J., Zhao, N., \& Zhu, T. (2020). The impact of COVID-19 epidemic declaration on psychological consequences: A study on active Weibo users. International Journal of Environmental Research and Public Health, 17, 2032. doi: 10.3390/ ijerph17062032 
McCann, I. L. \& Pearlman, L. A. (1990). Vicarious traumatization: A framework for understanding the psychological effects of working with victims. Journal of Traumatic Stress, 3(1), 131-149. doi: 10.1007/BF00975140

Mitchell, A. J., Yadegarfar, M., Gill, J., \& Stubbs, B. (2016). Case finding and screening clinical utility of the Patient Health Questionnaire (PHQ-9 and PHQ-2) for depression in primary care: a diagnostic meta-analysis of 40 studies. BJPsych Open, 2(2), 127-138. doi : 10.1192/bjpo.bp.115.001685

Morales-Chainé, S., López, M. A., Bosch, M. A., Beristain, A. A., Robles, G. R., et al. (en prensa). Condiciones de Salud Mental ante la contingencia sanitaria por COVID-19. Frontier.

Morales-Chainé, S., Rivera, B. A. M., Beristain, A. A., Cordera, T. T., López, M. A., Amaya, M. D., Bosh, M. A., Valero G. M. E., Méndez J. C. E., Becerril, R. C., Cuahonte, I. J. A., León, M. J., \& Cenobio, F. M. (2019). Salud integral en la comunidad universitaria: Detección temprana y psico-educación a distancia. En S., Morales-Chaine, A. López, L. S. Ortiz, P. G. Palafox, \& M. Malo. (Eds). Proyecto para superar las brechas de atención en salud mental mediante la implementación de la guía mhGAP en México. pp. 29-54. Organización Panamericana de la Salud.

Morales-Chainé, S., Palomero, J. C. A., Cuevas, R. C. M., Garibay, R., Palafox, P. G., \& Gómez, E. G. (en prensa). Estrés agudo en la segunda fase de la contingencia sanitaria COVID-19. Health and Addiction.

Moos, R. H. (1995). Development and applications of new measures of life stressors, social resources and coping responses. European Journal of Psychological Assessment, 11, 1-13. doi: 10.1027/1015-5759.11.1.1

Mortensen, C. R., Becker, D. V., Ackerman, J. M., Neuberg, S. L., \& Kenrich, D. T. (2010). The effects of disease salience on self-perceptions of personality and behavioral avoidance tendencies. Psychological. Science, 21(3), 440-447. doi: 10.1177/0956797610361706

Organización Mundial de la Salud. (2020) WHO Coronavirus Disease (COVID-19) Dashboard. https://covid19.who.int
Organización Panamericana de la Salud. (2020). Recuperado el 13 de abril de 2020 de https://who.maps.arcgis.com/apps/ webappviewer/index.html?id=2203b04c3a5f486685a15482aOd97a87\&extent $=-17277700.8881 \% 2 \mathrm{C}-1043174.5225 \% 2 \mathrm{C}-17$ 70156.5897\%2C6979655.9663\%2C102100

Secretaría de Salud en México. (2020). Recuperado el 9 de abril de 2020 de https://www.gob.mx/salud

Velasco, S., Ruiz, M. T., \& Álvarez-Dardet, C. (2006). Modelos de atención a los síntomas somáticos sin causa orgánica. De los trastornos fisiopatológicos al malestar de las mujeres (parte a). Revista Española de Salud Pública, 80(4) 317-322.

Wang, C., Pan, R., Wan, X., Tan, Y., Xu, L., Ho, C. S., \& Ho, R. C. (2020). Inmediate psychological responses and associated factors during the initial stage of the 2019 coronavirus disease (COVID-19) epidemic among the general population in China. International Journal of Environmental Research and Public Health, 17(5), 1729. doi: 10.3390/ijerph17051729

Xiang, Y. T., Yang, Y., Li, W., Zhang, L., Zhang, Q. Cheung, T., \& Ng, C. H. (2020). Timely mental health care for the 2019 novel coronavirus outbreak is urgently needed. The Lancet Psychiatry, 7(3), P228-229. doi: 10.1016/S2215-0366(20)30046-8

Yang, Y., Li, W., Zhang, Q., Zhang, L., Cheung, T., \& Xiang, Y.T. (2020). Mental health services for older adults in China during the COVID-19 outbreak. The Lancet Psychiatry, 7(4). doi: 10.1016/S2215-0366(20)30079-1

Zhang, M. W. B., Ho, C. S. H., Fang, P., Lu, Y., \& Ho, R. C. M. (2014). Methodology of developing a smartphone application for crisis research and its clinical application. Technology and Health Care, 22(4), 547-559. doi: 10.3233/THC-140819

Zandifar, A., \& Badrfam, R. (2020). Iranian mental health during the COVID-19 epidemic. Asian Journal of Psychiatry, 51. doi: 10.1016/j.ajp.2020.101990

Zhue, Y., Chen, L., Ji, H., Xi, M., Fang, Y., \& Li, Y. (2020). The risk and prevention of novel Coronavirus Pneumonia infections among inpatients in Psychiatric hospitals. Neuroscience Bulletin, 36, 299-302. doi: 10.1007/s12264-020-00476-9 